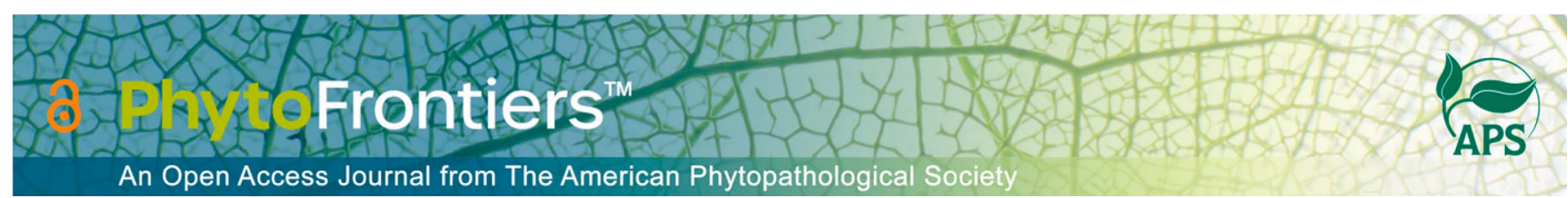

\title{
Research
}

\section{Monosaccharide Constituents of Potato Root Exudate Influence Hatching of the White Potato Cyst Nematode}

\author{
Christopher A. Bell (1) | Waddah Mobayed | Catherine J. Lilley | P. E. Urwin ${ }^{\dagger}$ |
}

Faculty of Biological Sciences, University of Leeds, Leeds, United Kingdom

\footnotetext{
† Corresponding author: P. E. Urwin; p.e.urwin@ leeds.ac.uk
}

Accepted for publication 14 February 2021.
Funding

Support was provided by the Council for At-Risk Academics.

The author(s) declare no conflict of interest.

\begin{abstract}
Plants secrete a large array of compounds into the rhizosphere to facilitate interactions with their biotic environment. Some of these exuded compounds stimulate the hatching of obligate plant-parasitic nematodes, ultimately leading to a detrimental effect on the host plant. Determining these cues can help to provide new mechanisms for control and aid nematode management schemes. Here, we show that glucose, fructose, and arabinose, which are all present in potato root exudate (PRE), induce hatching of white potato cyst nematode (Globodera pallida) eggs whereas five other PRE sugars had no effect. Although these monosaccharides resulted in significant hatching, none induced the same level as PRE, suggesting that other components, possibly in combination, contribute to stimulation of nematode hatching. Glucose but not arabinose or fructose was also observed to attract juvenile G. pallida, indicating that these hatch-inducing components can have different roles in different stages of the life cycle. Applying a solution of these monosaccharides to $G$. pallida-infested soil prepotato planting initiated hatching in the absence of a host. Host absence resulted in nematode mortality and a reduction in the G. pallida population. Therefore, subsequent invasion of the crop postplanting was also reduced compared with untreated soil. Our data suggest that monosaccharide components of PRE play an important role in the hatching and attraction of G. pallida. As a result, the hatch-inducing monosaccharides can be applied as a preplanting treatment to induce hatching and reduce subsequent infection rates.
\end{abstract}

Keywords: attraction, hatching, monosaccharides, potato cyst nematode, root exudate

Plant roots exude a wide array of compounds to facilitate complex and dynamic interactions with the rhizosphere, mediating both mutualistic and parasitic relationships (Barber and Martin 1976; Bertin et al. 2003; Canarini et al. 2019). Between 20 and $40 \%$ of fixed carbon is exuded from root tissue in the form of proteins, amino acids, 
carbohydrates, sugars, and organic acids, with the relative proportions of these constituents varying significantly between plant species (Bacilio-Jiménez et al. 2003; Badri and Vivanco 2009; Carvalhais et al. 2011; Kawasaki et al. 2016; Lugtenberg et al. 1999). Sugars represent approximately $75 \%$ (Vranova et al. 2013) of exuded material and include glucose, fructose, sucrose, arabinose, xylose, and galactose, all at varying concentrations (Badri and Vivanco 2009; Kawasaki et al. 2016). These concentrations also vary as the plant develops (Lugtenberg et al. 1999; Sasse et al. 2018), with total sugar exudation in Arabidopsis and rice sharply declining after 3 weeks of growth (Bacilio-Jiménez et al. 2003; Chaparro et al. 2013). In contrast, sucrose is only exuded from tomato roots that are $>4$ days old (Lugtenberg et al. 1999). Although several sugars are present, glucose represents over $90 \%$ of the carbohydrate content of root exudates of rice plants at 1 to 4 weeks of age (Bacilio-Jiménez et al. 2003). The regulation of compound exudation is a proposed mechanism for plant modulation of the rhizosphere (Micallef et al. 2009). This modulation is suggested to have evolved with the substrate specificity of plantassociated bacteria to assemble a root microbiome, which includes mutual, commensal, and parasitic interactions (Sasse et al. 2018). Plant-microbe relationships also impact plant root exudate profiles. For example, infection of Arabidopsis with Pseudomonas syringae reduces exudation of maltose, ribose, glucose, and fructose (Yuan et al. 2018).

Root exudates are vital mediators of interactions between plants and plant-parasitic nematodes. Globodera pallida is a highly evolved sedentary endoparasitic nematode that is commonly referred to as the white or pale potato cyst nematode (PCN) due to its prevalence on the crop and its restricted solanaceous host range. Eggs containing unhatched juvenile nematodes are protected in the soil within a cyst formed from the toughened body wall of the dead female. Each cyst contains approximately 400 eggs that can remain viable for decades before they begin hatching (Turner and Rowe 2006). The second-stage juvenile (J2) nematode hatches from the egg in response to root exudatederived cues in the soil and then penetrates the host root tissue. After migrating toward the vascular cylinder, a single cell is selected and modified by the nematode into a highly metabolically active feeding site, termed a syncytium. It is from this source that the nematode obtains nutrition to support development and subsequent egg laying (Lilley et al. 2005).

Hatching is a vital process in the life cycle of G. pallida and is historically thought to be initiated by certain compounds, termed hatching factors, present specifically in host root exudates. Hatching factors alter the permeability of the egg membrane, allowing the trehalose/water balance to shift, resulting in hydration of the juvenile and subsequent eclosion (Perry and Beane 1989). Solanoeclepin A, sodium thiocyanate, picloronic acid, $\alpha$-solanine, and $\alpha$-chaconine have been characterized as hatching factors for different cyst nematode species (Byrne et al. 2001; Schenk et al. 1999). In contrast to this assumed reliance on specific hatching factors, glucose and fructose were recently shown to induce hatch of G. pallida (Hoysted et al. 2018). This was unexpected due to their widespread presence in exudates from different plant species and the reported absolute requirement of host root exudate for hatching of G. pallida. The triggers of egg hatching are poorly characterized and a variety of components probably each contribute to the process. As well as affecting hatching, root exudates can induce stylet thrusting (Teillet et al. 2013), mediate attraction (Čepulytė et al. 2018; Phani et al. 2017), and stimulate gene expression responses (Bell et al. 2019) in plant-parasitic nematodes. Glucose and fructose induce attraction and stylet thrusting in $\mathrm{J} 2 \mathrm{~s}$ of the root-knot nematode Meloidogyne incognita but have no effect on those of G. pallida (Warnock et al. 2016). Changes in nematode gene expression are induced by carbohydrate components of root exudates, highlighting the complex interactions between nematodes and exudate carbohydrates (Bell et al. 2019).

The majority of research on exudate-nematode interactions has focused on motile nematode stages, with fewer studies investigating the effect of exudates on hatching. Here, we identify an additional monosaccharide, arabinose, which induces hatching of G. pallida, and determine the optimal concentrations of monosaccharides for hatch induction. We assayed each hatching-inducing compound for its role in attracting mobile nematodes, which plays an important role in multiple stages of the life cycle. Finally, we determined whether these compounds can be applied as a preplanting treatment to induce hatching in the absence of a host, thereby reducing the soil nematode population and the potential parasites of the subsequent crop.

\section{MATERIALS AND METHODS}

\section{Maintenance of plants and collection of root exudates}

Chitted tuber cuttings of Solanum tuberosum L. 'Désirée' were planted in $18-\mathrm{cm}$ pots containing sand and loam topsoil (50:50). Plants were grown in a glasshouse at 20 to $22^{\circ} \mathrm{C}$ under cycles of $16 \mathrm{~h}$ of light and $8 \mathrm{~h}$ of darkness for 3 weeks. Roots were excised intact from the bottom of the plant stem and washed to remove sand and loam. Roots were soaked in darkness for $24 \mathrm{~h}$ at $4^{\circ} \mathrm{C}$ at a concentration of $80 \mathrm{~g} /$ liter of tap water. This concentration of roots in water was chosen for its optimal induction of hatching and attraction of $G$. pallida (Farnier et al. 2012). The resultant potato root exudate (PRE) was sterilized using a $0.45-\mu \mathrm{m}$ filter and stored at $4^{\circ} \mathrm{C}$.

\section{Quantification of monosaccharides in PRE}

Root exudate was prepared from six individual root systems. The concentrations of arabinose, galactose, maltose, sucrose, and xylose were quantified colorimetrically at $340 \mathrm{~nm}$ using kits (K-MASUG and K-ARGA; Megazyme) according to the manufacturer's instructions. A mean concentration per biological replicate was calculated by measuring each PRE in technical triplicate. Water was used as a negative control and standards provided with the kits were used to construct calibration curves to quantify absorbance as micrograms per milliliter of each.

\section{G. pallida behavioral assays}

Hatching assay. For each hatching assay, batches of five $G$. pallida cysts were placed in wells of a 12-well polypropylene plate and $1 \mathrm{ml}$ of treatment solution was added, ensuring that the cysts were covered. Ten replicates of five cysts were carried out per treatment. Cysts were incubated at $20^{\circ} \mathrm{C}$ in treatment solution for 30 days, with the number of hatched juveniles counted and replacement with fresh solution every 5 days. Treatments consisted of either arabinose ( 2 to $162 \mu \mathrm{g} / \mathrm{ml}$ ), galactose $(20 \mu \mathrm{g} / \mathrm{ml})$, glucose ( 2 to $162 \mu \mathrm{g} / \mathrm{ml})$, fructose (4 to $315 \mu \mathrm{g} / \mathrm{ml})$, cellulose $(20 \mu \mathrm{g} / \mathrm{ml})$, maltose $(20 \mu \mathrm{g} / \mathrm{ml})$, lactose $(20 \mu \mathrm{g} / \mathrm{ml})$, sucrose $(20 \mu \mathrm{g} / \mathrm{ml})$, xylose $(20 \mu \mathrm{g} / \mathrm{ml})$, water (negative control), or PRE (positive control). After 30 days, the same cysts were washed in tap water and reincubated in PRE for a further 15 days. Each cyst was then opened and the number of unhatched $\mathrm{J} 2 \mathrm{~s}$ was counted to express the number of hatched $\mathrm{J} 2 \mathrm{~s}$ as a percentage of total potential hatch. 
Chemotaxis assay. Chemotaxis of G. pallida J2s toward sugars was assessed on 9-cm Petri plates containing 23\% (wt/ vol) Pluronic gel PF-127 (Anatrace). PF-127 was dissolved in water by continuous stirring for $24 \mathrm{~h}$ at $4{ }^{\circ} \mathrm{C}$, as described by Wang et al. (2009). The test solution was then pipetted $(20 \mu \mathrm{l})$ onto the test area of the plate and water was always added to the control area on the opposite side of the plate (Fig. 1). The optimal concentrations that induced hatching for glucose (18 $\mu \mathrm{g} / \mathrm{ml})$, fructose $(35 \mu \mathrm{g} / \mathrm{ml})$, and arabinose $(20 \mu \mathrm{g} / \mathrm{ml})$ were assayed for their attractiveness. Galactose was also assayed at $20 \mu \mathrm{g} / \mathrm{ml}$ as a nonhatch-inducing monosaccharide. Freshly hatched $\mathrm{J} 2 \mathrm{~s}(n=100)$ were washed multiple times in water and added to the center of the plate. The plate was then incubated in the dark at $20^{\circ} \mathrm{C}$ for $3 \mathrm{~h}$ before counting the number of nematodes within a $1-\mathrm{cm}$ radius of the test or control areas. The chemotaxis index (CI) (Hart 2006) was then calculated to show the relative chemotaxis of the nematodes toward or away from the treatment. For this index, $0<\mathrm{CI}<1$ is classed as attraction and $-1<\mathrm{CI}<0$ is classed as repulsion. Each treatment was assessed with five biological replicates.

\section{Glasshouse trial of monosaccharide control treatment}

Mesh bags were constructed to hold 20 cysts so that the hatched J2s could migrate through the mesh and the cysts could then be recovered from the soil. One bag containing 20 cysts was inserted into an $18-\mathrm{cm}$ pot containing sand and loam topsoil (50:50). The pot was saturated with approximately 150 $\mathrm{ml}$ of either PRE, a solution containing glucose and fructose (at 17 and $35 \mu \mathrm{g} / \mathrm{ml}$, respectively), or water. Each treatment was conducted with nine biological replicates. Pots were left for 3 weeks for hatching of eggs to occur, as dictated by the hatching assay data obtained previously. After 3 weeks, the cyst bags were removed from the soil and the number of unhatched eggs was counted. In a second experiment, this setup was repeated with five biological replicates; however, after the 3-week incubation, the pots were left in the glasshouse for a further 8 weeks to allow the death of any hatched juveniles (Robinson, et al. 1987). At this point, a chitted tuber was planted into each pot and grown for 3 weeks. Roots were then harvested, washed to remove soil, and stained with acid fuchsin to visualize the nematodes (Byrd et al. 1983). The number of nematodes in each root system was counted.

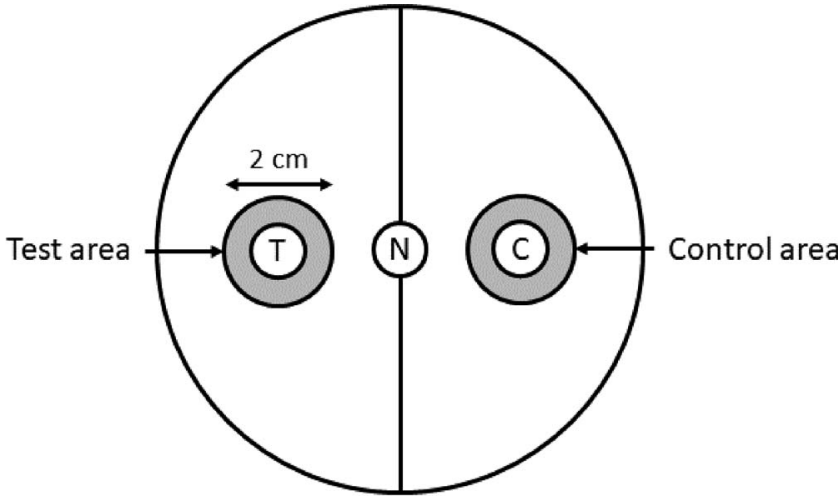

FIGURE 1

Schematic diagram of the chemotaxis assay plate. The test solution is pipetted into the test area $(T)$ and water is pipetted into the control area $(\mathrm{C})$. Nematodes are placed into the central circle $(\mathrm{N})$ and, after $3 \mathrm{~h}$, the nematodes within the 2-cm circle around each treated area (gray) are counted.

\section{Data analysis}

One-way analysis of variance (ANOVA) and StudentNewman-Keuls (SNK) post hoc tests were used to determine the significance of differences in G. pallida egg hatch, chemotaxis index, prepotato planting eggs per cyst and root invasion. SPSS v24 (IBM Corporation Armonk, New York, NY, U.S.A.) was used for all statistical analysis.

\section{RESULTS}

\section{Potato roots exude a range of mono- and disaccharides}

Assays were undertaken to determine potential G. pallida hatch-inducing sugars. Arabinose $(18.4 \pm 1.0 \mu \mathrm{g} / \mathrm{ml})$, galactose $(16.0 \pm 1.3 \mu \mathrm{g} / \mathrm{ml})$, maltose $(8.1 \pm 2.0 \mu \mathrm{g} / \mathrm{ml})$, and xylose $(22.3 \pm 1.5 \mu \mathrm{g} / \mathrm{ml}$ ) were detected in PRE (Fig. 2). Sucrose is important for carbon translocation in the plant but was not detected in PRE (Fig. 2).

\section{Glucose and fructose induce dose-dependent hatching of G. pallida}

Building on previous observations, we determined whether there were optimum concentrations of glucose and fructose that induced hatch of G. pallida. A range of both glucose and

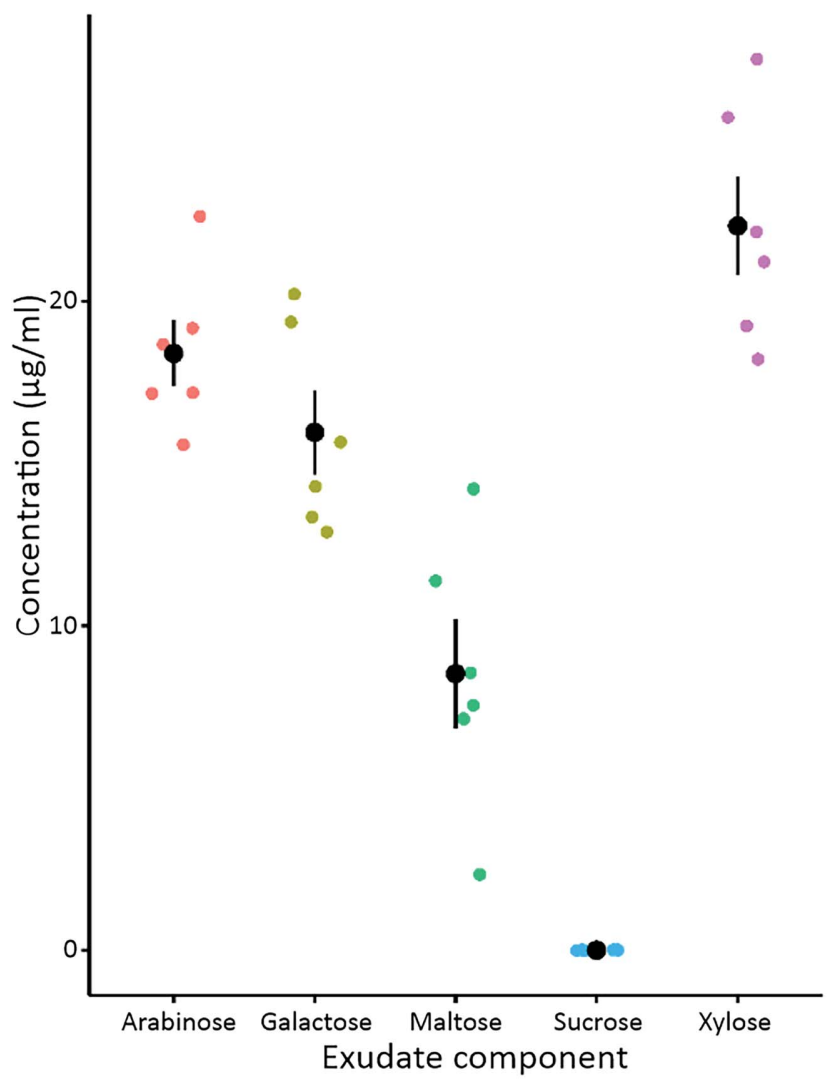

FIGURE 2

Potato root exudate contains multiple mono- and disaccharide components. Concentrations of arabinose, galactose, maltose, sucrose, and xylose present in potato root exudate. Concentrations were determined using K-MASUG and K-ARGA assay kits (Megazyme). Values are means \pm standard error of the mean from six replicates, with each representing a mean of three technical replicates. 
fructose concentrations induced significant hatching of G. pallida (Fig. 3A and B). Glucose induced significantly more hatch at $18 \mu \mathrm{g} / \mathrm{ml}$ than at the other concentrations; however, this was significantly lower than hatch induced by PRE (Fig. $3 \mathrm{~A})(P<0.05$, one-way ANOVA, SNK test). Higher concentrations of glucose at 162 and $54 \mu \mathrm{g} / \mathrm{ml}$ also induced significantly higher hatch than water $(P<0.05$, one-way ANOVA, SNK test) but lower hatch than $18 \mu \mathrm{g} / \mathrm{ml}$. The lowest concentrations of glucose $(6$ and $2 \mu \mathrm{g} / \mathrm{ml})$ did not induce any hatch.

Fructose at both 35 and $105 \mu \mathrm{g} / \mathrm{ml}$ (Fig. 3B) induced significantly higher hatch of $G$. pallida than water but lower than PRE over the 30-day assay period $(P<0.05$, one-way ANOVA, SNK test). Similar to glucose, the two lowest concentrations (12 and $4 \mu \mathrm{g} / \mathrm{ml}$ ) did not induce higher hatch than water but, in this case, the highest concentration of $315 \mu \mathrm{g} / \mathrm{ml}$ also did not induce significant hatching. In all assays, the viability and, thus, hatch potential of the eggs was established by replacing the treatments with PRE at day 30, resulting in an increase in hatching. Treatment with either sugar at any tested concentration resulted in a slower induction of hatch compared with PRE.

\section{Arabinose but not other sugars induces hatching of G. pallida}

The mono- and disaccharides detected in PRE, above, were then assayed for their effects on hatching of G. pallida. A concentration of $20 \mu \mathrm{g} / \mathrm{ml}$ was chosen to reflect the approximate amount of each detected in the PRE. Lactose was also assayed as a predicted negative control sugar because it is not described in the plant kingdom. Cellulose was included as an example of a polysaccharide present in plant root exudates (Bell et al. 2019). Arabinose treatment resulted in a significant hatching stimulus, albeit greatly reduced compare with PRE (Fig. 4A). The other saccharides had no effect on egg hatch. At day 30, all of the treatments were replaced with PRE, which established the hatching potential of the eggs. A range of concentrations of arabinose was then tested to determine an optimum hatching stimulus. Concentrations of 18 and $54 \mu \mathrm{g} /$ $\mathrm{ml}$ resulted in significantly higher hatching than water $(P<$ 0.05, one-way ANOVA, SNK test), albeit much lower than PRE, whereas arabinose at 2, 6, and $162 \mu \mathrm{g} / \mathrm{ml}$ did not significantly induce hatch (Fig. 4B).

\section{Glucose attracts J2s of G. pallida}

Hatched $\mathbf{J} 2$ nematodes must locate and move toward the host root in order to feed and reproduce. Therefore, the attractiveness of hatch-inducing sugars to G. pallida J2s was determined in agar plate assays. Nematodes migrated preferentially toward glucose solutions and PRE, with chemotaxis indexes of $0.24 \pm 0.08$ and $0.36 \pm 0.14$, respectively (Fig. 5) $(P<$ 0.01 , one-way ANOVA, SNK test). Fructose and arabinose, which induced nematode hatch, did not attract $\mathrm{J} 2 \mathrm{~s}$ of $\mathrm{G}$. pallida. Galactose, which was selected as a monosaccharide that did not induce hatching, also did not attract G. pallida J2s.

\section{Applying a glucose and fructose solution prepotato planting reduces the number of infective juveniles in the soil and subsequent root invasion}

The inducers of greatest $G$. pallida hatch, glucose and fructose, were assayed for their hatching stimulus in soil. Sterilized sandy loam soil containing cysts was saturated with either water, PRE, or a glucose/fructose solution. After 3 weeks, the cysts were recovered from the soil and significantly fewer unhatched encysted eggs were present after PRE and glucose/fructose treatments, compared with water $(P<0.05$, one-way ANOVA, SNK test) (Fig. 6A). The treatments were then repeated and the soil was left for 8 weeks in the glasshouse to ensure death of any hatched juveniles in the absence of a host plant. A potato tuber was then planted into each pot and the number of invaded nematodes in each root system was counted after 3 weeks. Significantly reduced root invasion was observed in potato plants grown in soil previously treated with PRE or glucose/fructose solution $(P<0.05$, one-way ANOVA, SNK test) (Fig. 6B).

\section{DISCUSSION}

Here, we determined the optimal concentrations of monosaccharides that induce hatching of G. pallida eggs and attraction of infective juveniles. Notably, we utilized these components as a preplanting control for the parasitic nematode, whereby they induced hatching in the absence of a host, resulting in a reduced soil population available for infection of the subsequent crop. This approach exploits monosaccharides that are naturally exuded by host potato roots to combat an economically important parasitic nematode.

Exudates from plant roots vary in composition and concentrations between plant species (Bacilio-Jiménez et al. 2003; Carvalhais et al. 2011; Kawasaki et al. 2016; Lugtenberg et al. 1999). Root exudates from host plant species are widely known to stimulate hatching and attraction of cyst nematodes (Devine et al. 1996; Farnier et al. 2012) as well as inducing stylet thrusting (Teillet et al. 2013), attraction (Čepulytè et al. 2018; Phani et al. 2017), and transcriptional responses (Bell et al. 2019) in other plant-parasitic nematodes. Specifically, glucose and fructose are known to induce hatching of $G$. pallida and attraction and stylet thrusting of $M$. incognita (Hoysted et al. 2018; Warnock et al. 2016). Content of these sugars and expression of sugar transporter proteins were found to increase in leaves and roots of tomato during infection by $M$. incognita (Zhao et al. 2018). Bacterial and fungal pathogens are also known to induce the expression of different sugar transporter genes (Chen et al. 2010), suggesting that pathogens target these genes for nutritional gain. In this study, both glucose and fructose were found to induce hatch of G. pallida at varying concentrations; however, 18 and 105 or $35 \mu \mathrm{g} / \mathrm{ml}$, respectively, were the optima. It is possible that the optimal fructose concentration may be between 105 and $35 \mu \mathrm{g} / \mathrm{ml}$ or that there is a broader range for this monosaccharide's effect on hatching. Reduced hatching observed under lower concentrations suggests that a threshold must be reached before hatching is initiated. Interestingly, higher concentrations also resulted in reduced hatch, suggesting a possible feedback mechanism and a concentration-response relationship. Alternatively, it could reflect the fine osmotic balance that is required for egg hatching. J2s of G. pallida are partially dehydrated within the egg, to restrict movement and prolong energy reserves and survival (Perry 2002). The permeability of the eggshell changes upon stimulation from PRE, allowing trehalose to leak out concurrently with an influx of water, reducing osmotic pressure of the J2, which then hydrates and can perforate and exit the egg (Perry 2002; Wright and Perry 2006). Therefore, the osmotic pressure of the solution to which the egg is exposed may have a significant impact on the readiness for trehalose to leak from within the egg and, therefore, induce hatch. The osmotic strength of the 


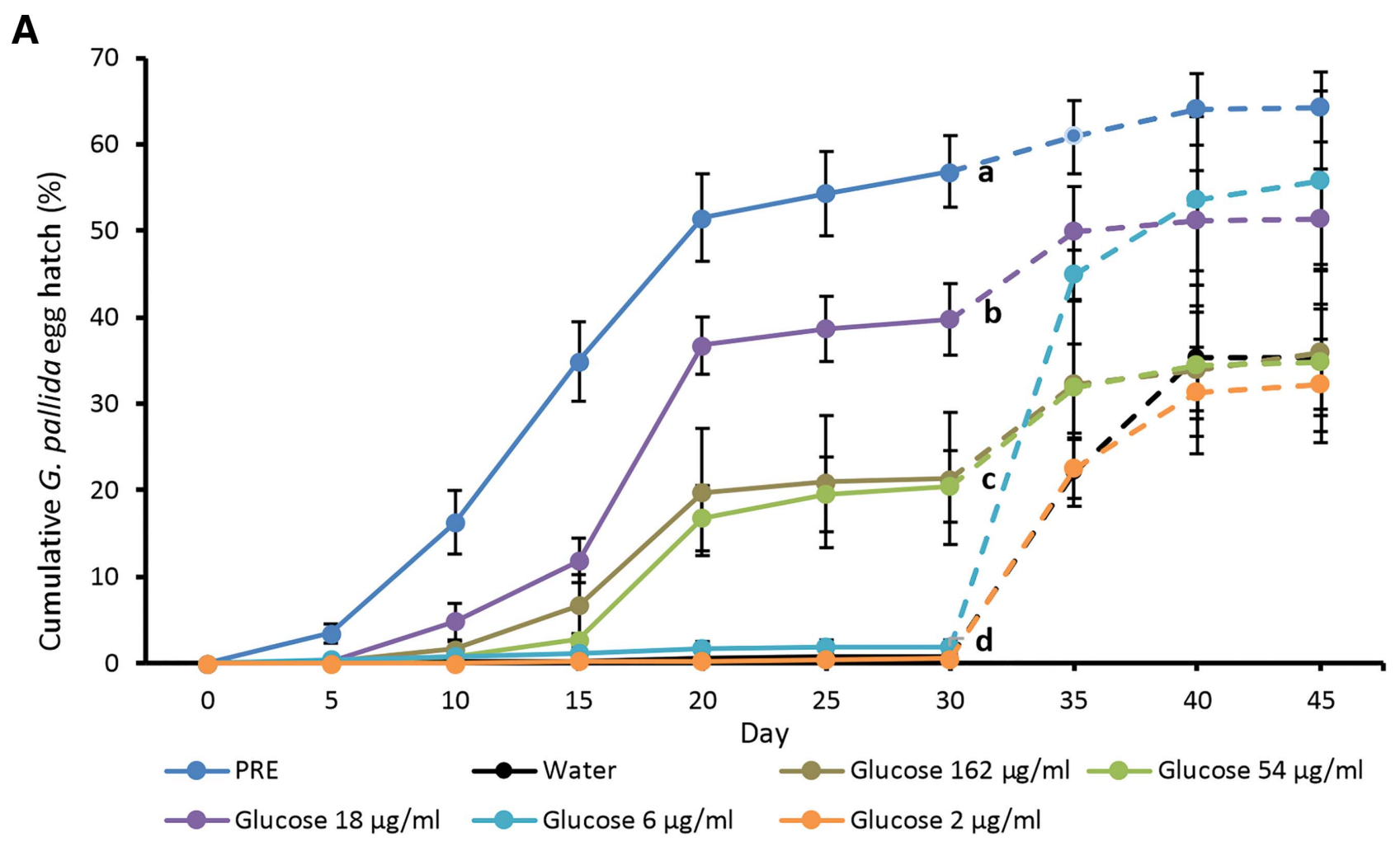

B

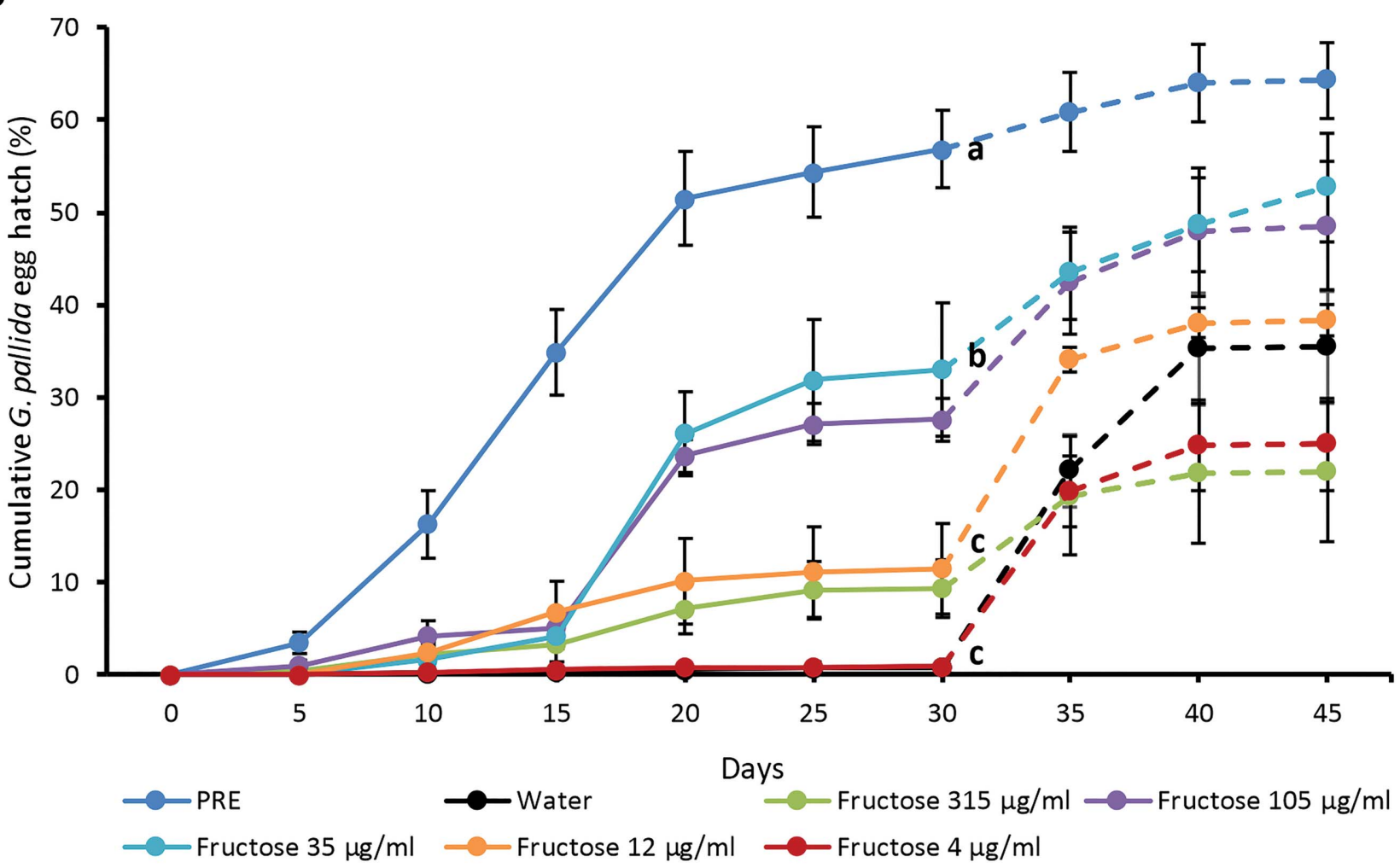

FIGURE 3

Concentrations of glucose and fructose differentially induce hatching of Globodera pallida. Concentrations of A, glucose at 2, 6, 18, 54, and $162 \mu \mathrm{g} / \mathrm{ml}$ and $\mathbf{B}$, fructose at $4,12,35,105$, and $315 \mu \mathrm{g} / \mathrm{ml}$ were applied to cysts (days 0 to 30 ) and cumulative percent egg hatch was recorded. Potato root exudate (PRE) was applied to all cysts during days 30 to 45 (dashed line) as a known inducer of hatch, establishing the hatching viability of all cysts. Values are means \pm standard error of the mean from 10 replicates, with five cysts per replicate. Different letters denote significance of cumulative hatching values at day $30(P<0.05$, one-way analysis of variance, Student-NewmanKeuls test). 


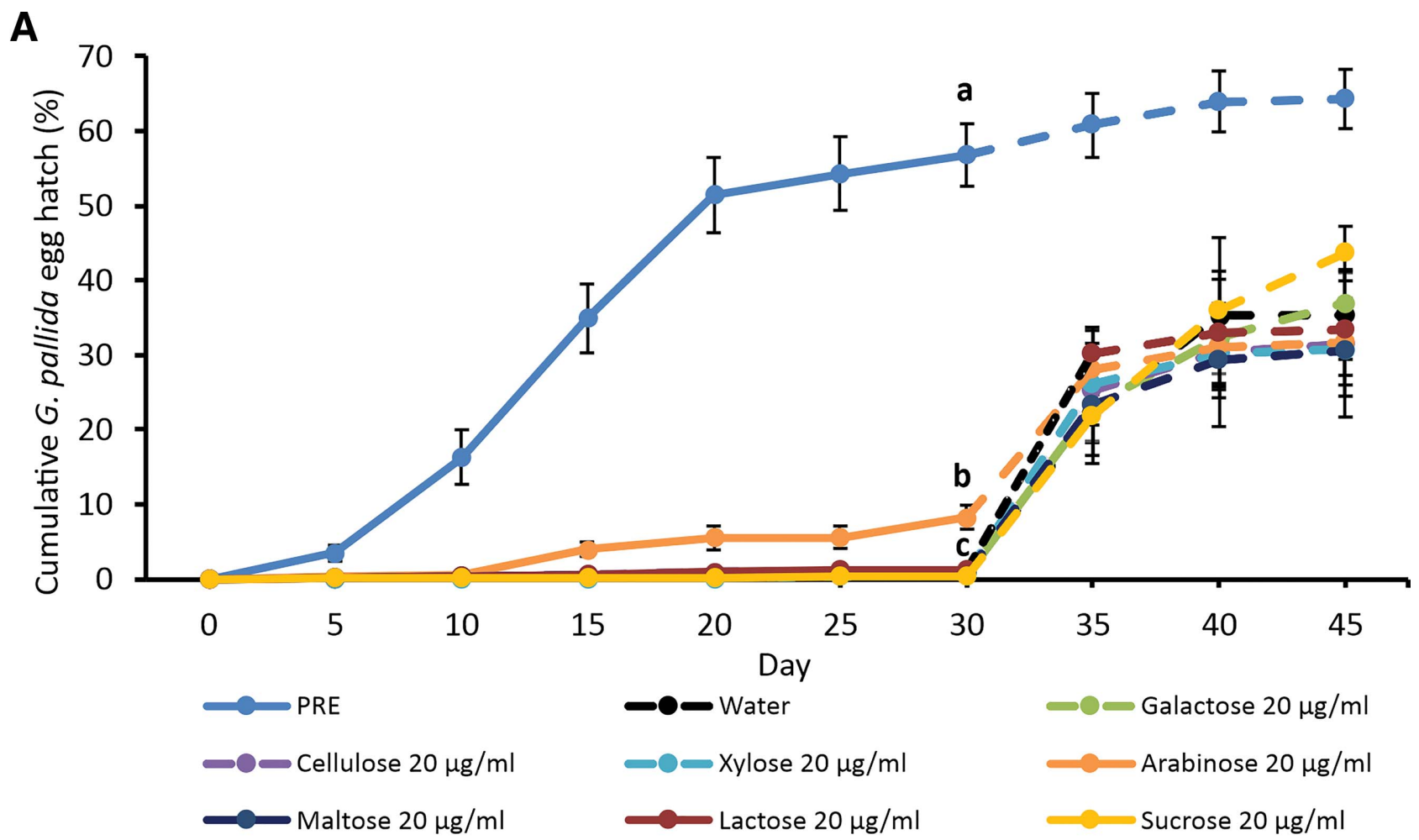

B

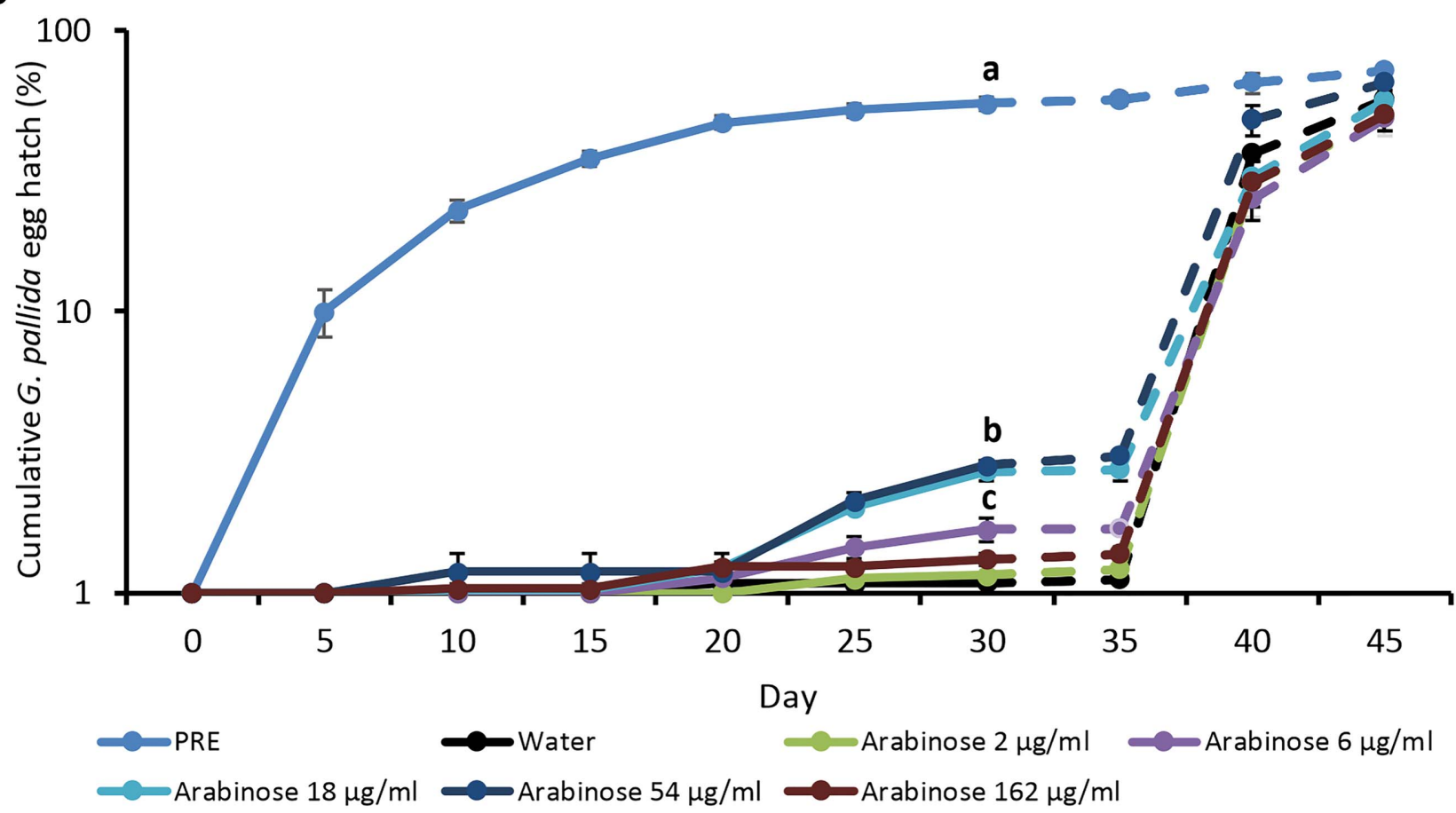

FIGURE 4

Arabinose induces hatching of Globodera pallida. A, Arabinose, cellulose, galactose, maltose, lactose, sucrose, and xylose were applied to cysts at single concentrations to assess stimulation of hatch. B, Concentrations of arabinose at $2,6,18,54$, and $162 \mu \mathrm{g} / \mathrm{ml}$ were applied to cysts to determine the optimum for induction of hatch. Potato root exudate (PRE) was applied to all cysts during days 30 to 45 (dashed line) as a known inducer of hatch, establishing the hatching viability of all cysts. Values are means \pm standard error of the mean from 10 replicates, with five cysts per replicate. Different letters denote significance of cumulative hatching values at day $30(P<0.05$ one-way analysis of variance, Student-Newman-Keuls test). 
higher concentrations of monosaccharides applied in this study may restrict water entering the egg and, therefore, result in less hatch. It would be of interest to investigate the osmotic pressure of eggs within different solutions and also throughout the time of exposure. Similar to our data, higher concentrations of PRE reduce the emergence of cyst nematode juveniles from eggs, also indicating a fine balance of parameters are required for nematode hatch (Devine et al. 1996; Hague 1958).

Nematode chemoreception toward roots is a vital component of parasitism and G. pallida are known to be attracted toward PRE (Farnier et al. 2012). Here, we found that glucose attracts G. pallida $\mathrm{J} 2 \mathrm{~s}$ at the same rate as PRE. The lack of attraction toward fructose and arabinose, coupled with the hatch induction from the monosaccharides, indicates that different components are involved in different aspects of the parasitism process. Interestingly, a previous study observed attraction of $M$. incognita, but not G. pallida toward glucose and fructose (Warnock et al. 2016). The contrasting results from our study may be due to different attraction assay methodology as well as the different populations of $G$. pallida, because cyst populations are genetically distinct (Plantard et al. 2008).

Analysis of PRE identified the additional presence of arabinose, galactose, maltose, and xylose but not sucrose, which was unexpected, because sucrose is an abundant carbohydrate in

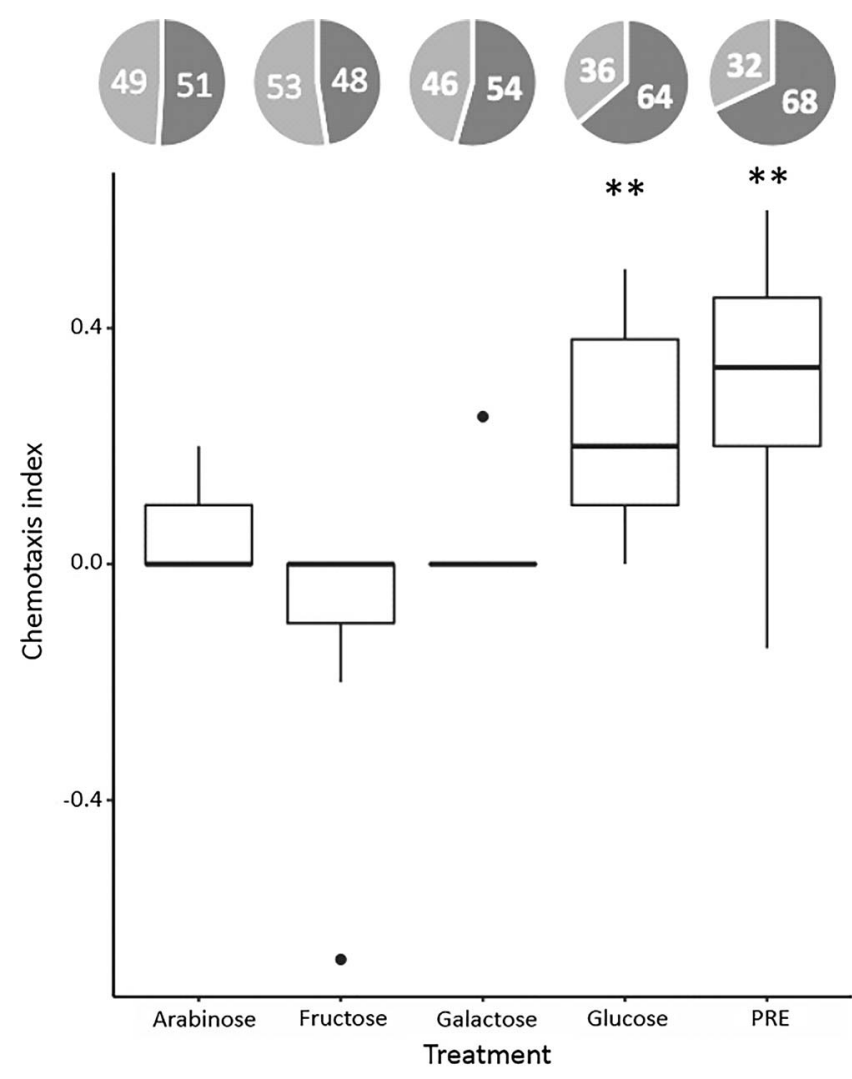

FIGURE 5

The hatch-inducing sugar glucose also attracts Globodera pallida juveniles. Attraction of $G$. pallida second-stage juveniles was measured toward arabinose, galactose, glucose, fructose, and potato root exudate (PRE) after $3 \mathrm{~h}$ of exposure. Pie charts display the mean percentage of nematodes in the treatment (darker) or control (lighter) zones of the plate. Water was used as the control attractant in each setup with 100 nematodes used per replicate. Black circles represent outliers on the boxplots. Asterisks denote significantly more nematodes in the treatment area compared with control area from five replicates $(P<0.01$, one-way analysis of variance, Student-Newman-Keuls test). plants that is known to be exuded (Kawasaki et al. 2016; Naher et al. 2009). Of these sugars, only arabinose (at 18 and $54 \mu \mathrm{g} / \mathrm{ml}$ ) induced nematode hatch, although at a much lower rate than glucose, fructose, or PRE. Arabinose is transported between plant cells (Rottmann et al. 2018) and, therefore, could be acquired and utilized by $G$. pallida while feeding, similar to the transport of glucose into the syncytia and female cyst nematodes (Hofmann et al. 2009). If so, the monosaccharides studied here may be exudate signals to the egg that conditions are suitable for hatching and that resources are nearby. This could be in addition to the factors that are known to be required for the egg to end diapause and hatch (Turner and Rowe 2006). Cysts were exposed to PRE after the sugar assays to confirm that the differences in egg hatching were a result of the treatment and not due to reduced viability of the selected cysts. The difference in the extent of this recovery between treatments suggests that either the saccharides may have a deleterious effect on a proportion of eggs or the prolonged preincubation in nonoptimal hatching conditions lowers subsequent hatch. The mechanisms behind this are not currently known.

The induction of hatching by sugars was tested for its potential as a preplanting control treatment by reducing the number of G. pallida in the soil. By treating soil with a oneoff dose of glucose and fructose, there was a significant reduction in the number of viable eggs when cysts were recovered. Furthermore, if the soil was then left for 8 weeks before planting of a tuber, significantly fewer nematodes invaded the roots in the pots treated with the monosaccharide solution. This indicates that earlier hatching had occurred and was followed by death of the $G$. pallida $\mathrm{J} 2$ s once their stored energy reserves had been exhausted in the absence of a host plant. In each instance, PRE provided similar results; however, the efficacy of collecting enough exudate would be unfeasible in a field situation compared with the monosaccharide solution. Several control measures aim at reducing egg hatch while inducing nematode mortality (Jung et al. 2002; Siddiqui and Shaukat 2003; Wei et al. 2014); however, inducing the early emergence of juveniles from eggs in the absence of a host plant is an appealing alternative. Trap crops such as S. sisymbriifolium produce root exudates that stimulate hatching of PCN and allow their invasion into the root; however, the plants' defense responses lead to degeneration of the nematode feeding structures (Varandas et al. 2020). This inhibits PCN reproduction and reduces the field population. Our data suggest that this approach could potentially be taken further, by inducing hatch of $G$. pallida eggs by applying soil treatments rather than planting a second crop. They could also be applied postharvest to reduce the population prior to the subsequent crop; however, this may not be successful for species such as G. pallida, which need a diapause before hatching can occur. Further studies are needed to scale up applications; however, the logistics of a soil treatment compared with the planting and harvesting of a trap crop seem beneficial. Incorporating tomato root exudates into soil can reduce the number of viable G. pallida eggs per cyst by up to $79 \%$ (Devine and Jones 2001), while the exogenous application of hatching factors to field soil can reduce the population size of G. rostochiensis by approximately 50\% (Devine and Jones 2000). This approach avoids the application of compounds that are lethal to the nematodes and potentially to other organisms; however, collecting exudates or hatching factors is an intensive task. Although we did not observe any microbial growth on the surface of the soil from the sugar treatments, monosaccharides are known to stimulate soil microbial activity and accelerate decomposition 

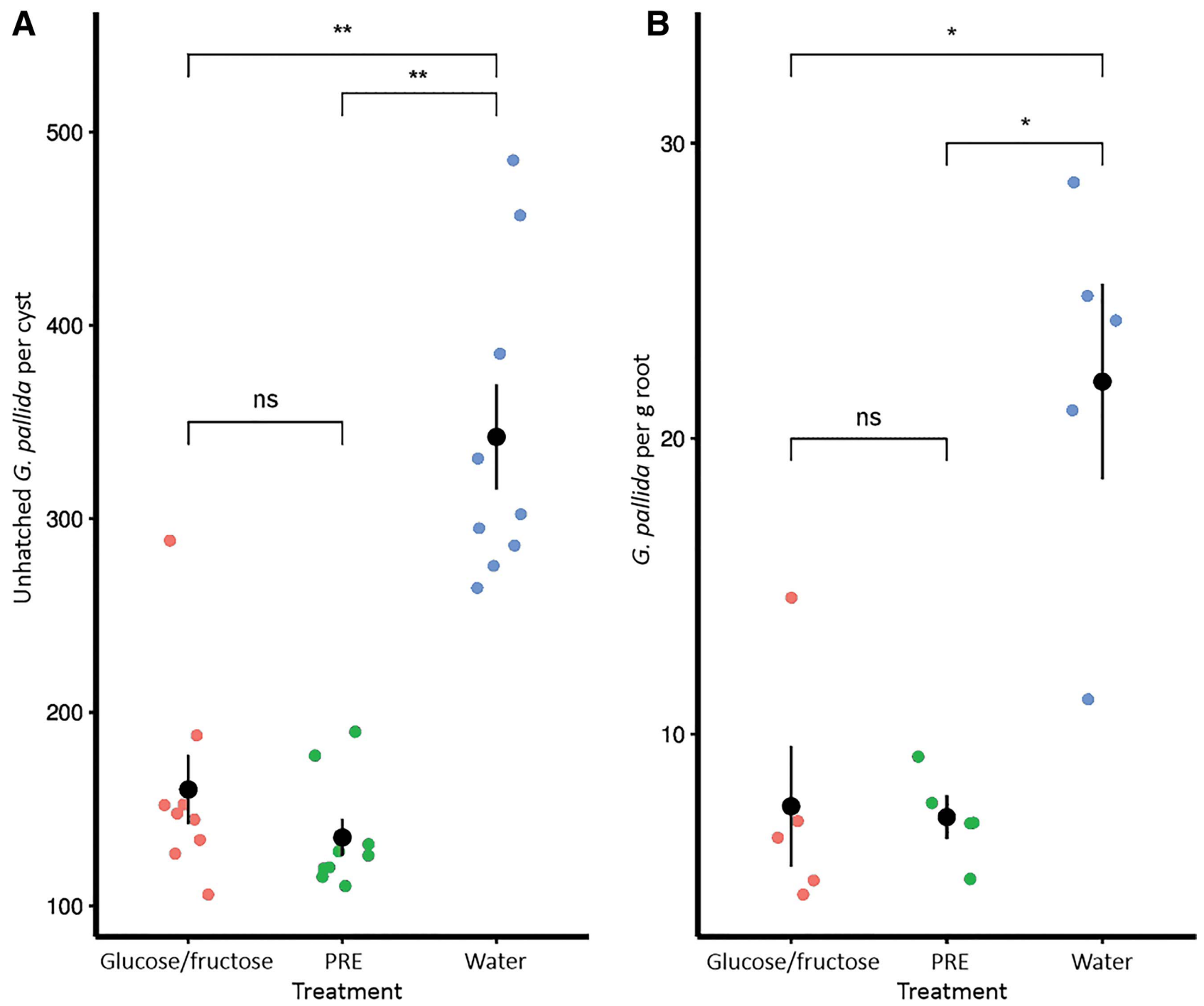

FIGURE 6

A, Prepotato planting treatment of cyst-containing soil with glucose and fructose induces hatching of Globodera pallida and B, results in reduced root invasion of subsequent crop. Soil containing cysts was saturated with a solution of glucose and fructose (17 and $35 \mu \mathrm{g} / \mathrm{ml}$, respectively), potato root exudate (PRE), or water, then incubated at $20^{\circ} \mathrm{C}$ for 3 weeks before cyst recovery, and the number of unhatched eggs was counted. In a separate experiment, soil containing cysts was treated as above and then left for 8 weeks for hatching and subsequent death of hatched juveniles to occur. Potato tubers were then planted in these pots and root invasion by freshly hatched juveniles quantified after 3 weeks of growth. Black circles represent the means \pm standard error of the mean from nine (A) and five (B) replicates, with 20 cysts per replicate. Asterisks denote significant differences between treatments $(*$ and $* *$ indicate $P<0.05$ and 0.01 , respectively, and $\mathrm{ns}=$ not significant, one-way analysis of variance, Student-Newman-Keuls test).

in the rhizosphere (Blagodatskaya and Kuzyakov 2013; Gunina and Kuzyakov 2015). Soils with varying microbiota do not affect the hatching potential of PRE on G. pallida eggs (Gautier et al. 2020); however, rigorous testing is needed to ensure that application of these components does not shift the soil community with knock-on effects on the crop. However, these effects may not necessarily be negative; glucose amendments to the soil surface can suppress pathogens and enhance seedling growth (Shimizu et al. 2018).

\section{LITERATURE CITED}

Bacilio-Jiménez, M., Aguilar-Flores, S., Ventura-Zapata, E., Pérez-Campos, E., Bouquelet, S., and Zenteno, E. 2003. Chemical characterization of root exudates from rice (Oryza sativa) and their effects on the chemotactic response of endophytic bacteria. Plant Soil 249:271-277.
Badri, D. V., and Vivanco, J. M. 2009. Regulation and function of root exudates. Plant Cell Environ. 32:666-681.

Barber, D. A., and Martin, J. K. 1976. The release of organic substances by cereal roots into soil. New Phytol. 76:69-80.

Bell, C. A., Lilley, C. J., McCarthy, J., Atkinson, H. J., and Urwin, P. E. 2019. Plant-parasitic nematodes respond to root exudate signals with host-specific gene expression patterns. PLoS Pathog 15:e1007503.

Bertin, C., Yang, X., and Weston, L. A. 2003. The role of root exudates and allelochemicals in the rhizosphere. Plant Soil 256:67-83.

Blagodatskaya, E., and Kuzyakov, Y. 2013. Active microorganisms in soil: Critical review of estimation criteria and approaches. Soil Biol. Biochem. 67:192-211.

Byrd, D., Kirkpatrick, T., and Barker, K. 1983. An improved technique for clearing and staining plant-tissues for detection of nematodes. J. Nematol. 15:142-143.

Byrne, J. T., Maher, N. J., and Jones, P. W. 2001. Comparative responses of Globodera rostochiensis and G. pallida to hatching chemicals. J. Nematol. 33:195-202. 
Canarini, A., Kaiser, C., Merchant, A., Richter, A., and Wanek, W. 2019. Root exudation of primary metabolites: Mechanisms and their roles in plant responses to environmental stimuli. Front. Plant Sci. 10:157.

Carvalhais, L. C., Dennis, P. G., Fedoseyenko, D., Hajirezaei, M. R., Borriss, R., and Von Wirén, N. 2011. Root exudation of sugars, amino acids, and organic acids by maize as affected by nitrogen, phosphorus, potassium, and iron deficiency. J. Plant Nutr. Soil Sci. 174:3-11.

Čepulyte, R., Danquah, W. B., Bruening, G., and Williamson, V. M. 2018. Potent attractant for root-knot nematodes in exudates from seedling root tips of two host species. Sci. Rep. 8:10847.

Chaparro, J. M., Badri, D. V., Bakker, M. G., Sugiyama, A., Manter, D. K., and Vivanco, J. M. 2013. Root exudation of phytochemicals in Arabidopsis follows specific patterns that are developmentally programmed and correlate with soil microbial functions. PLoS One 8.

Chen, L.-Q., Hou, B.-H., Lalonde, S., Takanaga, H., Hartung, M. L., Qu, X.-Q., Guo, W.-J., Kim, J.-G., Underwood, W., Chaudhuri, B., Chermak, D., Antony, G., White, F. F., Somerville, S. C., Mudgett, M. G., and Frommer, W. B. 2010. Sugar transporters for intercellular exchange and nutrition of pathogens. Nature 468:527-532.

Devine, K. J., Byrne, J., Maher, N., and Jones, P. W. 1996. Resolution of natural hatching factors for golden potato cyst nematode, Globodera rostochiensis. Ann. Appl. Biol. 129:323-334.

Devine, K. J., and Jones, P. W. 2000. Response of Globodera rostochiensis to exogenously applied hatching factors in soil. Ann. Appl. Biol. 137:21-29.

Devine, K. J., and Jones, P. W. 2001. Effects of hatching factors on potato cyst nematode hatch and in-egg mortality in soil and in vitro. Nematology 3:65-74.

Farnier, K., Bengtsson, M., Becher, P. G., Witzell, J., Witzgall, P., and Manduríc, S. 2012. Novel bioassay demonstrates attraction of the white potato cyst nematode Globodera Pallida (Stone) to non-volatile and volatile host plant cues. J. Chem. Ecol. 38:795-801.

Gautier, C., Martinez, L., Fournet, S., Montarry, J., Yvin, J.-C., NguemaOna, E., Guillerm-Erckelboudt, A.-Y., Piriou, C., Linglin, J., Mougel, C., and Lebreton, L. 2020. Hatching of Globodera pallida induced by root exudates is not influenced by soil microbiota composition. Front. Microbiol. 11:2489.

Gunina, A., and Kuzyakov, Y. 2015. Sugars in soil and sweets for microorganisms: Review of origin, content, composition and fate. Soil Biol. Biochem. 90:87-100.

Hague, N. G. 1958. The concentration of potato root diffusate under reduced pressure. Nematologica 3:149-153.

Hart, A. C. 2006. Behaviour. In: WormBook: The Online Review of C. elegans Biology. The C. elegans Research Community, ed. https:// www.ncbi.nlm.nih.gov/books/NBK19734/

Hofmann, J., Hess, P. H., Szakasits, D., Blöchl, A., Wieczorek, K., Daxböck-Horvath, S., Bohlmann, H., Van Bel, A. J. E., and Grundler, F. M. W. 2009. Diversity and activity of sugar transporters in nematode-induced root syncytia. J. Exp. Bot. 60:3085-3095.

Hoysted, G. A., Bell, C. A., Lilley, C. J., and Urwin, P. E. 2018. Aphid colonization affects potato root exudate composition and the hatching of a soil borne pathogen. Front. Plant Sci. 9:1278.

Jung, W. J., Jung, S. J., An, K. N., Jin, Y. L., Park, R. D., Kim, K. Y., Shon, B. K., and Kim, T. H. 2002. Effect of chitinase-producing Paenibacillus illinoisensis KJA-424 on egg hatching of root-knot nematode (Meloidogyne incognita). J. Microbiol. Biotechnol. 12:865871 .

Kawasaki, A., Donn, S., Ryan, P. R., Mathesius, U., Devilla, R., Jones, A., and Watt, M. 2016. Microbiome and exudates of the root and rhizosphere of Brachypodium distachyon, a model for wheat. PLoS One 11:e0164533.

Lilley, C. J., Atkinson, H. J., and Urwin, P. E. 2005. Molecular aspects of cyst nematodes. Mol. Plant Pathol. 6:577-588.

Lugtenberg, B. J., Kravchenko, L. V., and Simons, M. 1999. Tomato seed and root exudate sugars: Composition, utilization by Pseudomonas biocontrol strains and role in rhizosphere colonization. Environ. Microbiol. 1:439-446.

Micallef, S. A., Shiaris, M. P., and Colón-Carmona, A. 2009. Influence of Arabidopsis thaliana accessions on rhizobacterial communities and natural variation in root exudates. J. Exp. Bot. 60:1729-1742.
Naher, U. A., Othman, R., Mohd Saud, H., Shamsuddin, Z., and Ismail, M. R. 2009. Influence of root exudate carbon compounds of three rice genotypes on rhizosphere and endophytic diazotrophs. Pertanika J. Trop. Agric. Sci. 32:209-223.

Perry, R. N. 2002. Hatching. Pages 147-169 in: The Biology of Nematodes. D. L. Lee, ed. Taylor and Francis, London, U.K.

Perry, R. N., and Beane, J. 1989. Effects of certain herbicides on the in vitro hatch of Globodera rostochiensis and Heterodera schachtii. Rev. Nématol. 12:191-196.

Phani, V., Shivakumara, T. N., Davies, K. G., and Rao, U. 2017. Meloidogyne incognita fatty acid- and retinol-binding protein (Mi-FAR-1) affects nematode infection of plant roots and the attachment of Pasteuria penetrans endospores. Front. Microbiol. 8:2122.

Plantard, O., Picard, D., Valette, S., Scurrah, M., Grenier, E., and Mugniéry, D. 2008. Origin and genetic diversity of Western European populations of the potato cyst nematode (Globodera pallida) inferred from mitochondrial sequences and microsatellite loci. Mol. Ecol. 17:2208-2218.

Robinson, M. P., Atkinson, H. J., and Perry, R. N. 1987. The influence of temperature on the hatching activity and lipid utilization of second stage juveniles of the potato cyst nematodes Globodera rostochiensis and G. pallida. Rev. Nématol. 10:349-354.

Rottmann, T., Klebl, F., Schneider, S., Kischka, D., Rüscher, D., Sauer, N., and Stadler, R. 2018. Sugar transporter STP7 specificity for Larabinose and D-xylose contrasts with the typical hexose transporters STP8 and STP12. Plant Physiol. 176:2330-2350.

Sasse, J., Martinoia, E., and Northen, T. 2018. Feed your friends: Do plant exudates shape the root microbiome? Trends Plant Sci. 23:25-41.

Schenk, H., Driessen, R. A. J., De Gelder, R., Goubitz, K., Nieboer, H., Brüggemann-Rotgans, I. E. M., and Diepenhorst, P. 1999. Elucidation of the structure of solanoeclepin A. a natural hatching factor of potato and tomato cyst nematodes, by single-crystal X-ray diffraction. Croat. Chem. Acta 72:593-606.

Shimizu, Y., Sagiya, D., Matsui, M., and Fukui, R. 2018. Zonal soil amendment with simple sugars to elevate soil $\mathrm{C} / \mathrm{N}$ ratios as an alternative disease management strategy for rhizoctonia damping-off of sugar beet. Plant Dis. 102:1434-1444.

Siddiqui, I. A., and Shaukat, S. S. 2003. Suppression of root-knot disease by Pseudomonas fluorescens CHA0 in tomato: Importance of bacterial secondary metabolite, 2.,4-diacetylpholoroglucinol. Soil Biol. Biochem. $35: 1615-1623$

Teillet, A., Dybal, K., Kerry, B. R., Miller, A. J., Curtis, R. H. C., and Hedden, P. 2013. Transcriptional changes of the root-knot nematode Meloidogyne incognita in response to Arabidopsis thaliana root signals. PLoS One 8:e61259.

Turner, S. J., and Rowe, J. A. 2006. Cyst nematodes. Pages 91-122 in: Plant Nematology. R. N. Perry and M. Moens, eds. CABI, Wallingford, U.K

Varandas, R., Egas, C., and Conceicao, I. L. 2020. Potato cyst nematodes: New solutions to an old problem. Crop Prot. 137:105303.

Vranova, V., Rejsek, K., Skene, K. R., Janous, D., and Formanek, P. 2013. Methods of collection of plant root exudates in relation to plant metabolism and purpose: A review. J. Plant Nutr. Soil Sci. 176:175-199.

Wang, C., Lower, S., and Williamson, V. M. 2009. Application of Pluronic gel to the study of root-knot nematode behaviour. Nematology 11:453-464.

Warnock, N. D., Wilson, L., Canet-Perez, J. V., Fleming, T., Fleming, C. C., Maule, A. G., and Dalzell, J. J. 2016. Exogenous RNA interference exposes contrasting roles for sugar exudation in hostfinding by plant pathogens. Int. J. Parasitol. 46:473-477.

Wei, L., Shao, Y., Wan, J., Feng, H., Zhu, H., Huang, H., and Zhou, Y. 2014. Isolation and characterization of a rhizobacterial antagonist of root-knot nematodes. PLoS One 9:e85988.

Wright, D. J., and Perry, R. N. 2006. Reproduction, physiology and biochemistry. Pages 187-209 in: Plant Nematology. R. N. Perry and M. Moens, eds. CABI, Wallingford, U.K.

Yuan, J., Zhao, J., Wen, T., Zhao, M., Li, R., Goossens, P., Huang, Q., Bai, Y., Vivanco, J. M., Kowalchuk, G. A., Berendsen, R. L., and Shen, Q. 2018. Root exudates drive the soil-borne legacy of aboveground pathogen infection. Microbiome 6:156.

Zhao, D., You, Y., Fan, H., Zhu, X., Wang, Y., Duan, Y., Xuan, Y., and Chen, L. 2018. The role of sugar transporter genes during early infection by root-knot nematodes. Int. J. Mol. Sci. 19:302. 